\title{
Abstracts from Nippon Eiseigaku Zasshi (Japanese Journal of Hygiene) vol. 63 no. 3
}

Published online: 5 August 2008

(C) The Japanese Society for Hygiene 2008

\section{Review of the Approach to Exercise Behavior Modification from the Viewpoint of Preventive Medicine}

Nippon Eiseigaku Zasshi, 63, 617-627 (2008)

Takuo Nomura ${ }^{1}$, Munetsugu Kouta ${ }^{2}$, Kenta Shigemori ${ }^{3}$,

Yoshinobu Yoshimoto ${ }^{4}$ and Atsushi Sato ${ }^{5}$

${ }^{1}$ Department of Physical Therapy, School of Comprehensive

Rehabilitation, Osaka Prefecture University

${ }^{2}$ Department of Rehabilitation, Hiroshima City General

Rehabilitation Center

${ }^{3}$ Department of Physical Therapy, School of Rehabilitation

Sciences, Seirei Christopher University

${ }^{4}$ Department of Rehabilitation Medicine, Koseinenkin

Kochi Rehabilitation Hospital

${ }^{5}$ Department of Health Science, Faculty of Human Life

and Environmental Science, Kochi Women's University

The purpose of this study was to summarize the approaches to behavior modification for exercise from the viewpoint of preventive medicine. Articles were searched according to the particular field of preventive medicine, i.e., primary prevention, secondary prevention, tertiary prevention, and other fields of prevention. In the field of primary prevention for elderly people living at home, many fall prevention programs were found to have been carried out. In these studies, various programs were found to be effective if the exercise proved to be sufficient. Although some approaches were observed to be based on the productive aging theory and social capital, the number of such studies was small. In the field of secondary prevention, illness and functional disorders are prevented from becoming worse. It is therefore important for each individual to exercise by himself/herself and also acquire sufficient self-monitoring skills. Social capital is useful for learning good exercise habits. In the field of tertiary prevention, although exercise therapy is effective for improving physical functions and preventing disease recurrence in patients with chronic disease, some patients nevertheless find it difficult to continue such an exercise therapy. The approaches to behavior modification were extremely effective for patients with chronic disease. In other fields of preventive medicine, daily exercises such stair climbing are effective methods for reducing the risk of chronic disease and such a behavior modification may lead to a considerable public health gain. In the future, further studies with a many lines of evidence should be performed, and approaches based on behavioral science should be established.

\section{Oxidative Stress in Porphyria and Carriers}

Nippon Eiseigaku Zasshi, 63, 628-635 (2008)

Masahito Aminaka ${ }^{1}$, Masao Kondo ${ }^{2}$, Ayako Takata ${ }^{1}$,

Hiroshi Yamauchi ${ }^{3}$, Maki Ikeda ${ }^{3}$ and Katsumi Yoshida ${ }^{1}$

${ }^{1}$ Department of Preventive Medicine, St. Marianna University

School of Medicine

${ }^{2}$ Toyoko Gakuen Women's College

${ }^{3}$ Department of Public Health, School of Alled Health Science, Kitasato University

We sought to establish a causal relationship between oxidative stress and porphyria in patients and carriers. We reported changes in urinary porphyrin concentrations related to 8-hydroxy-2'-deoxyguanosine.

Methods: We measured urinary 8-hydroxy-2'-deoxyguanosine concentration in porphyria patients and carriers with multifactorial inheritance as a possible marker of attack. The porphyria types included 10 patients with porphyria cutanea tarda, 5 with variegate porphyria, 8 with hereditary coproporphyria, 7 with congenital erythropoietic porphyria, 5 with erythropoietic protoporphyria, 5 with acute intermittent porphyria, 7 erythropoietic protoporphyria carriers, and 7 acute intermittent porphyria carriers.

Results: Urinary porphyrin concentrations in these patients were significantly higher than those in healthy subjects $(P<0.001)$. Urinary 8-hydroxy-2'-deoxyguanosine concentrations were significantly high in dermatopathy porphyria types namely porphyria cutanea tarda $(P<0.001)$, variegate porphyria $(P<0.05)$, hereditary coproporphyria $(P<0.05)$, congenital erythropoietic phyria $(P<0.05)$, and erythropoietic protoporphyria $(P<0.001)$.

Conclusion: These results reveal that urinary 8-hydroxy-2'-deoxyguanosine concentration in cutis porphyria types is a good predictor of attack and abatement. 


\section{Relationships of Self-Reported Physical Health, Sociability, and Spiritual Life with Mental Health: An Investigation According to Gender and Life Stage}

Nippon Eiseigaku Zasshi, 63, 636-641 (2008)

Yukie Kumagai ${ }^{1}$, Ikuharu Morioka ${ }^{2}$, Kouichi Yoshimasu ${ }^{1}$, Hiroe Tomita $^{3}$, Nobuyuki Miyai ${ }^{1,4}$ and Kazuhisa Miyashita ${ }^{1}$

${ }^{1}$ Department of Hygiene, School of Medicine,

Wakayama Medical University

${ }^{2}$ School of Health and Nursing Science,

Wakayama Medical University

${ }^{3}$ Health Promotion Division in Wakayama Prefecture

${ }^{4}$ Osaka Kyoiku University

Objective: To investigate the relationships of self-reported physical health, social and spiritual satisfactions with subjective mental health according to gender and life stage.

Methods: Self-reported questionnaires with questions regarding subjective health status and lifestyles were sent to 1,905 community residents aged 20 years or older selected by random sampling in Wakayama Prefecture, Japan. Subjective mental health, physical health, social, and spiritual satisfactions as measures of subjective health status were assessed using a visual analogue scale. Multiple logistic regression analysis was used to evaluate the relationships between the subjective mental health and the other three components of the subjective health status with adjustment for life style factors related to the subjective mental health in the subjects without missing values of relevant factors $(n=1,752)$. The subjects were divided into three life stages depending on their ages: young (20-39), middle (4059 ), and old (60 or more).

Results: Overall, the poor statuses of the three components, namely, physical health, social and spiritual satisfactions, were significantly associated with poor mental health in both genders at any life stage. However, detailed analysis revealed some interesting characteristics. In young men, social dissatisfaction was strongly associated with poor mental health whereas spiritual dissatisfaction in addition to social dissatisfaction was strongly associated with poor mental health in young women. In the middle aged group, both social and spiritual dissatisfactions were strongly associated with poor mental health in both genders. On the other hand, poor physical health status was strongly associated with poor mental health in the old aged group. Conclusions: These results suggest the importance of the mental health interventions under consideration for each life stage and gender.

\section{Effects of Resistance Training on Physical Fitness, Muscle Strength, and Natural Killer Cell Activity in Female University Students}

Nippon Eiseigaku Zasshi, 63, 642-650 (2008)

Tamami Takahashi ${ }^{1}$, Yoshihiro Arai ${ }^{2}$, Michiko Hara ${ }^{3}$,

Kihachi Ohshima $^{4}$, Sakuji Koya ${ }^{5}$ and Tetsuro Yamanishi ${ }^{2}$

${ }^{1}$ Gunma University

${ }^{2}$ Department of Health and Sports Science,

Faculty of Education, Gunma University

${ }^{3}$ Department of Education of Handicapped Children,

Faculty of Education, Gunma University

${ }^{4}$ University Health Care Center, Gunma University

${ }^{5}$ Wakanyaku Medical Institute, Ltd.

Objectives: The health benefits of resistance training for women have been well documented. However, few investigations have been reported on the immune responses to resistance training among young people. To determine the effects of resistance training on natural killer cell activity (NKCA) in young people, a study was conducted among healthy female university students.

Methods: The study was conducted with 22 healthy nonathlete female university students (aged $19.8 \pm 1.3$ years) who volunteered to participate in the study. Six women were assigned to exercise group (a) that carried out resistance training five or six times a week, another six women were assigned to exercise group (b) that carried out resistance training three or four times a week, and the remaining ten comprised, the control group (nonexercise group). The exercise groups carried out resistance training for both the upper and lower parts of the body using ankle and wrist weights for 8 weeks. Blood samples were obtained at the onset of the study (before the start of any activity in the exercise groups) and at the conclusion of the training (8 weeks later) in order to determine NKCA. A physical fitness test and a muscle strength test were conducted on the subjects to assess the strength of the upper and lower parts of the body, muscular endurance, and flexibility.

Results: The mean NKCA at the conclusion of the training showed a significant increase in exercise group (b), even though there was no significant difference in NKCA in the nonexercise group or in exercise group (a) between before and after the training. An improvement in physical fitness and muscle strength was observed in exercise group (a) and exercise group (b).

Conclusions: This study showed that resistance training improved physical fitness, muscle strength, and NKCA in young female subjects. Regarding the effects of exercise frequency on NKCA, this study suggests that exercise carried out three of four times a week might be associated with an increase in NKCA.

\section{Study of Clarifying Incidence of Lifestyle-Related Diseases and Related Complications and Its Medical Expenses Using Government-Managed Health Insurance Data}

Nippon Eiseigaku Zasshi, 63, 651-661 (2008)

Hiroyuki Sakamaki ${ }^{1}$, Takefumi Kitazawa ${ }^{2}$ and Takashi Muto ${ }^{3}$

${ }^{1}$ Meijo University, Faculty of Pharmaceutical Science

${ }^{2}$ Toho University, School of Medicine

${ }^{3}$ Dokkyo Medical University, School of Medicine

Objectives: In this study, analysis was performed with the objective of clarifying the incidence of lifestyle-related diseases and related complications and its medical expenses.

Methods: A random sample population of people insured under government-managed health insurance in Mie Prefecture, Japan, who had undergone annual health examinations in 1993, 1998, and 2003, and who had incurred medical costs in 2003 was analyzed. The relationship between the results of health examinations in 1993 and medical costs in 2003 was investigated by examining 2,165 individuals whose data were available. The relationship between medical costs and risk factors including diabetes, hypertension, hyperlipemia, cerebrovascular disease, and ischemic heart disease was examined by analysis of covariance adjusted by sex and age.

Results: There was a higher doctor consultation rate for diabetes, hypertension, and hyperlipemia after 10 years among people presenting with high Body Mass Index, blood pressure, serum lipid, and plasma glucose.

Conclusions: It was shown that there is a relationship of having lifestyle-related diseases and related complications with doctor consultation rate and medical expenditure. 


\section{Health Effects of Nanoparticles and Nanomaterials (II) Methods for Measurement of Nanoparticles and Their Presence in the Air}

Nippon Eiseigaku Zasshi, 63, 663-669 (2008)

Yuji Fujitani and Seishiro Hirano

Environmental Nanotoxicology Section, RCER,

National Institute for Environmental Studies

The mass concentrations of airborne particles in the atmospheric, indoor, and industrial environments are regulated by air quality standards. Epidemiological studies show that there are significant positive correlations between particle mass concentrations and adverse health effects. In these context nanoparticles in the air, which are defined as particles with a diameter (Dp) of less than $50 \mathrm{~nm}$ or $100 \mathrm{~nm}$ for engineered ones, are gaining increasing attention despite a small contribution to the mass of total airborn particles. Contrary to the mass concentration the number concentrations of atmospheric nanoparticles are quite high in most cases. Moreover there is limited toxicological information on nanoparticles, although the deposition rate of nanoparticles in the respiratory region is known to be relatively high. Accordingly there are a lot of debates about what metric is best to depict the size distribution of nanoparticles, number, surface area, or mass. In this paper, we report methods for measurement of nanoparticles on the basis of those metrics.

We also report sources of nanoparticle in the environment and occupational settings. The high number concentration of nanoparticles of $20-30 \mathrm{~nm}$ modal diameters have been documented at roadsides. Diesel-powered vehicles are major sources of those nanoparticles in the urban atmosphere. Engineered nanoparticles generate in some occupational settings in the handling processes such as bagging and cleaning with vacuum cleaners. 